\title{
Regular and chaotic dynamics in time-dependent relativistic mean-field theory
}

\author{
D. Vretenar*, P. Ring, G. A. Lalazissis and W. Pöschl \\ Physik-Department der Technischen Universität München, \\ D-85748 Garching, Germany
}

October 30, 2018

\begin{abstract}
Isoscalar and isovector monopole oscillations that correspond to giant resonances in spherical nuclei are described in the framework of time-dependent relativistic mean-field theory. Time-dependent and self-consistent calculations that reproduce experimental data on monopole resonances in ${ }^{208} \mathrm{~Pb}$ show that the motion of the collective coordinate is regular for isoscalar oscillations, and that it becomes chaotic when initial conditions correspond to the isovector mode. Regular collective dynamics coexists with chaotic oscillations on the microscopic level. Time histories, Fourier spectra, state-space plots, Poincaré sections, autocorrelation functions, and Lyapunov exponents are used to characterize the nonlinear system and to identify chaotic oscillations. Analogous considerations apply to higher multipolarities.
\end{abstract}

\footnotetext{
${ }^{1}$ Alexander von Humboldt Fellow, on leave of absence from University of Zagreb, Croatia
} 


\section{Introduction}

In the last two decades many studies have been reported in which the atomic nucleus has been used as a laboratory, both experimentally and theoretically, for investigating the transition from order to chaos in quantum dynamical systems (for a recent review see [1]). Most of these studies have concentrated on two major aspects: (i) generic signatures of chaos in local fluctuations and correlations of nuclear level distributions, and (ii) chaos in microscopic and collective dynamics of realistic many-body systems. In the first case, signatures of quantum chaos have also been studied in the complicated structure of wave functions and randomness of matrix elements of physical operators. On the other hand, the nature of collective nuclear dynamics has been investigated with particular emphasis on the stability of low-lying nuclear modes in relation to one-body dissipation caused by the deformation of the nuclear potential, cluster effects and Coriolis forces. The most elementary collective modes in nuclei are giant resonances. These are highly collective nuclear excitations in which a large fraction of nucleons participate. They can be described as damped harmonic/anharmonic density oscillations around the equilibrium ground-state. Giant resonances occur over the whole periodic table and their characteristic parameters are smooth functions of the mass number. A mean-field model therefore provides an appropriate framework for the description of giant resonances. Regular and chaotic dynamics in giant nuclear oscillations has been the subject of a number of studies. What has emerged as a very interesting result is that an undamped collective mode may coexist with chaotic single-particle motion. It appears that the slowly vibrating selfconsistent mean field created by the nucleons averages out the random components in their motion. In all investigations the motion of only one type of particles has been considered. That is, only the dynamics of isoscalar collective modes. 
In the present article we study the difference in the dynamics of isoscalar and isovector collective modes. In particular, we describe isoscalar and isovector monopole oscillations in spherical nuclei, but analogous considerations apply to higher multipolarities. The dynamics of nuclear vibrations is analyzed in the framework of time-dependent relativistic mean-field theory (TD RMFT). The model represents a relativistic generalization of the time-dependent Hartree-Fock approach. Nuclear dynamics is described by the simultaneous evolution of $A$ single particle Dirac spinors in the time-dependent mean fields. Frequencies of eigenmodes are determined from a Fourier analysis of dynamical quantities. In this microscopic description, self-consistent mean-field calculations are performed for static ground-state properties, and time-dependent calculations for monopole excitations. Because the time-evolution is calculated self-consistently, the system is intrinsically nonlinear. An advantage of the time-dependent approach is that no assumption about the nature of the mode of vibrations has to be made.

The article is organized as follows. In Sec. 2 we present the essential features of the time-dependent relativistic mean-field model, as well as some details of its application to spherical nuclei. Time-dependent calculations of isoscalar and isovector monopole oscillations in ${ }^{16} \mathrm{O}$ and ${ }^{208} \mathrm{~Pb}$ are described in Sec. 3. Results of a number of diagnostic

tests that are used to identify chaotic oscillations in the nuclear system are discussed and compared with earlier work. A summary of our results is presented in Sec. 4.

\section{Outline of the model}

The dynamics of collective vibrations in spherical nuclei will be described in the framework of relativistic mean-field theory [2, 匀, 河. In relativistic quantum hadrodynamics the nucleus is described as a system of Dirac nucleons which interact through the exchange 
of virtual mesons and photons. The Lagrangian density of the model is

$$
\begin{aligned}
\mathcal{L}= & \bar{\psi}(i \gamma \cdot \partial-m) \psi+\frac{1}{2}(\partial \sigma)^{2}-U(\sigma) \\
& -\frac{1}{4} \Omega_{\mu \nu} \Omega^{\mu \nu}+\frac{1}{2} m_{\omega}^{2} \omega^{2}-\frac{1}{4} \overrightarrow{\mathrm{R}}_{\mu \nu} \overrightarrow{\mathrm{R}}^{\mu \nu}+\frac{1}{2} m_{\rho}^{2} \vec{\rho}^{2}-\frac{1}{4} \mathrm{~F}_{\mu \nu} \mathrm{F}^{\mu \nu} \\
& -g_{\sigma} \bar{\psi} \sigma \psi-g_{\omega} \bar{\psi} \gamma \cdot \omega \psi-g_{\rho} \bar{\psi} \gamma \cdot \vec{\rho} \vec{\tau} \psi-e \bar{\psi} \gamma \cdot A \frac{\left(1-\tau_{3}\right)}{2} \psi
\end{aligned}
$$

The Dirac spinor $\psi$ denotes the nucleon with mass $m . m_{\sigma}, m_{\omega}$, and $m_{\rho}$ are the masses of the $\sigma$-meson, the $\omega$-meson, and the $\rho$-meson, and $g_{\sigma}, g_{\omega}$, and $g_{\rho}$ are the corresponding coupling constants for the mesons to the nucleon. $U(\sigma)$ denotes the nonlinear $\sigma$ selfinteraction

$$
U(\sigma)=\frac{1}{2} m_{\sigma}^{2} \sigma^{2}+\frac{1}{3} g_{2} \sigma^{3}+\frac{1}{4} g_{3} \sigma^{4}
$$

and $\Omega^{\mu \nu}, \vec{R}^{\mu \nu}$, and $F^{\mu \nu}$ are field tensors [2].

The coupled equations of motion are derived from the Lagrangian density (西). The Dirac equation for the nucleons:

$$
\begin{aligned}
i \partial_{t} \psi_{i}= & {\left[\boldsymbol{\alpha}\left(-i \boldsymbol{\nabla}-g_{\omega} \boldsymbol{\omega}-g_{\rho} \vec{\tau} \overrightarrow{\boldsymbol{\rho}}-e \frac{\left(1-\tau_{3}\right)}{2} \boldsymbol{A}\right)+\beta\left(m+g_{\sigma} \sigma\right)\right.} \\
& \left.+g_{\omega} \omega_{0}+g_{\rho} \vec{\tau} \vec{\rho}_{0}+e \frac{\left(1-\tau_{3}\right)}{2} A_{0}\right] \psi_{i}
\end{aligned}
$$

and the Klein-Gordon equations for the mesons:

$$
\begin{aligned}
\left(\partial_{t}^{2}-\Delta+m_{\sigma}^{2}\right) \sigma & =-g_{\sigma} \rho_{s}-g_{2} \sigma^{2}-g_{3} \sigma^{3} \\
\left(\partial_{t}^{2}-\Delta+m_{\omega}^{2}\right) \omega_{\mu} & =g_{\omega} j_{\mu} \\
\left(\partial_{t}^{2}-\Delta+m_{\rho}^{2}\right) \vec{\rho}_{\mu} & =g_{\rho} \vec{j}_{\mu} \\
\left(\partial_{t}^{2}-\Delta\right) A_{\mu} & =e j_{\mu}^{\mathrm{em}} .
\end{aligned}
$$

In the relativistic mean field approximation the A nucleons described by single-particle spinors $\psi_{i},(i=1,2, \ldots, A)$, are assumed to form the A-particle Slater determinant $|\Phi\rangle$, 
and to move independently in the classical meson fields. The sources of the fields, i.e. densities and currents, are calculated in the no-sea approximation [7]: - the scalar density

$$
\rho_{\mathrm{s}}=\sum_{i=1}^{A} \bar{\psi}_{i} \psi_{i}
$$

- the isoscalar baryon current

$$
j^{\mu}=\sum_{i=1}^{A} \bar{\psi}_{i} \gamma^{\mu} \psi_{i},
$$

- the isovector baryon current

$$
\vec{j}^{\mu}=\sum_{i=1}^{A} \bar{\psi}_{i} \gamma^{\mu} \vec{\tau} \psi_{i},
$$

- the electromagnetic current for the photon-field

$$
j_{\mathrm{em}}^{\mu}=\sum_{i=1}^{A} \bar{\psi}_{i} \gamma^{\mu} \frac{1-\tau_{3}}{2} \psi_{i},
$$

where the summation is over all occupied states in the Slater determinant $|\Phi\rangle$. Negativeenergy states do not contribute to the densities in the no-sea approximation of the stationary solutions. However, negative energy contributions are included implicitly in the time-dependent calculation, since the Dirac equation is solved at each step in time for a different basis set [6, 7]. Negative energy components with respect to the original ground state basis are taken into account automatically, even if at each time the no-sea approximation is applied. It is also assumed that nucleon single-particle states do not mix isospin.

The ground state of a nucleus is described by the stationary self-consistent solution of the coupled system of equations (3)-(7), for a given number of nucleons and a set of coupling constants and masses. The solution for the ground state specifies part of the initial conditions for the time-dependent problem. For a given set of initial conditions, i.e. initial values for the densities and currents in Eqs. (8 11), the model describes the time evolution of $A$ single-particle wave functions in the time-dependent mean fields. 
Retardation effects for the meson fields are not included, i.e. the time derivatives $\partial_{t}^{2}$ in the equations of motions for the meson fields are neglected. This is justified by the large masses in the meson propagators, causing a short range of the corresponding meson exchange forces. Since there is no systematic procedure to derive the initial conditions that characterize the motion of a specific mode of the nuclear system, the description of nuclear dynamics as a time-dependent initial-value problem is intrinsically semi-classical. The theory can be quantized by the requirement that there exist time-periodic solutions of the equations of motion, which give integer multiples of Planck's constant for the classical action along one period [8]. For giant resonances the time-dependence of collective dynamical quantities is actually not periodic, since generally giant resonances are not stationary states of the mean-field Hamiltonian. The coupling of the mean-field to the particle continuum allows for the decay of giant resonances by direct escape of particles. In the limit of small amplitude oscillations, however, the energy obtained from the frequency of the oscillation coincides with the excitation energy of the collective state. In Refs. [7, 9, 8, 11] we have shown that the model reproduces reasonably well the experimental data on giant resonances in spherical nuclei.

In the present article we apply the model to isoscalar and isovector monopole oscillations in spherical nuclei. In this microscopic description, self-consistent mean-field calculations are performed for static ground-state properties, and time-dependent calculations for monopole excitations. Because of the self-consistent time-evolution, the system is intrinsically non-linear. For a system with spherical symmetry, the nucleon single-particle spinor is characterized by the angular momentum $j$, its $z$-projection $m$, the parity $\pi$, and the isospin $t_{3}= \pm \frac{1}{2}$ for neutrons and protons

$$
\psi\left(\mathbf{r}, t, s, t_{3}\right)=\frac{1}{r}\left(\begin{array}{c}
f(r) \Phi_{l j m}(\theta, \varphi, s) \\
i g(r) \Phi_{\tilde{l j m}}(\theta, \varphi, s)
\end{array}\right) e^{-i E t} \chi_{\tau}\left(t_{3}\right) .
$$


$\chi_{\tau}$ is the isospin function, the orbital angular momenta $l$ and $\tilde{l}$ are determined by $j$ and the parity $\pi, f(r)$ and $g(r)$ are radial functions, and $\Phi_{l j m}$ is the tensor product of the orbital and spin functions

$$
\Phi_{l j m}(\theta, \varphi, s)=\sum_{m_{s} m_{l}}<\frac{1}{2} m_{s} l m_{l} \mid j m>Y_{l m_{l}}(\theta, \varphi) \chi_{m_{s}}(s) .
$$

In order to excite monopole oscillations, the spherical solution for the ground-state has to be initially compressed or radially expanded by scaling the radial coordinate. The amplitudes $f^{\text {mon }}$ and $g^{\text {mon }}$ of the Dirac spinor are defined

$$
f^{\mathrm{mon}}\left(r_{\mathrm{mon}}\right)=(1+a) f(r), \quad g^{\mathrm{mon}}\left(r_{\mathrm{mon}}\right)=(1+a) g(r)
$$

The new coordinates are

$$
r_{\text {mon }}=(1+a) r
$$

For isoscalar oscillations the monopole deformations of the proton and neutron densities have the same sign. To excite isovector oscillations, the initial monopole deformation parameters of protons and neutrons must have opposite signs. After the initial deformation (14), the proton and neutron densities have to be normalized.

The time-dependent Dirac equation (3) is reduced to a set of coupled first-order partial differential equations for the complex amplitudes $f$ and $g$ of proton and neutron states

$$
\begin{aligned}
i \partial_{t} f & =\left(V_{0}+g_{\sigma} \sigma\right) f+\left(\partial_{r}-\frac{\kappa}{r}-i V_{r}\right) g \\
i \partial_{t} g & =\left(V_{0}-g_{\sigma} \sigma-2 m\right) g-\left(\partial_{r}+\frac{\kappa}{r}-i V_{r}\right) f
\end{aligned}
$$

where $\kappa= \pm\left(j+\frac{1}{2}\right)$ for $j=l \mp \frac{1}{2}$, and the indices 0 and $r$ denote the time and radial components of the vector field

$$
V^{\mu}=g_{\omega} \omega^{\mu}+g_{\rho} \rho_{3}^{\mu} \tau_{3}+e \frac{\left(1-\tau_{3}\right)}{2} A^{\mu}
$$


For a given set of initial conditions, the equations of motion propagate the nuclear system in time. The potentials are solutions of the Klein-Gordon equations

$$
\left(-\frac{\partial^{2}}{\partial r^{2}}-\frac{2}{r} \frac{\partial}{\partial r}+m_{\phi}^{2}\right) \phi(r)=s_{\phi}(r),
$$

$m_{\phi}$ are meson masses for $\phi=\sigma, \omega$ and $\rho$, and zero for the photon. The source terms are calculated from (8)-(11) using in each time-step the latest values for the nucleon amplitudes. The meson fields and electromagnetic potentials are calculated from

$$
\phi(r)=\int_{0}^{\infty} G_{\phi}\left(r, r^{\prime}\right) s_{\phi}\left(r^{\prime}\right) r^{\prime 2} d r^{\prime}
$$

where for massive fields

$$
G_{\phi}\left(r, r^{\prime}\right)=\frac{1}{2 m_{\phi}} \frac{1}{r r^{\prime}}\left(e^{m_{\phi}\left|r-r^{\prime}\right|}-e^{-m_{\phi}\left|r+r^{\prime}\right|}\right)
$$

and for the Coulomb field

$$
\begin{aligned}
& G_{C}\left(r, r^{\prime}\right)=\frac{1}{r} \quad \text { for } r>r^{\prime} \\
& G_{C}\left(r, r^{\prime}\right)=\frac{1}{r^{\prime}} \quad \text { for } r<r^{\prime} .
\end{aligned}
$$

The collective dynamical variables that characterize vibrations of a nucleus are defined as expectation values of single-particle operators in the time-dependent Slater determinant $|\Phi(t)\rangle$ of occupied states. In the framework of the TDRMF model the wave-function of the nuclear system is a Slater determinant at all times. For isoscalar monopole vibrations, the time-dependent monopole moment is defined:

$$
\left\langle r^{2}(t)\right\rangle=\frac{1}{A}\left\langle\Phi(t)\left|r^{2}\right| \Phi(t)\right\rangle
$$

The corresponding isovector monopole moment is simply

$$
<r_{\mathrm{p}}^{2}(t)>-<r_{\mathrm{n}}^{2}(t)>
$$

Fourier transforms of the collective dynamical variable determine the frequencies of eigenmodes. 


\section{Isoscalar and isovector monopole oscillations}

The study of isoscalar monopole resonances in nuclei provides an important source of information on the nuclear matter compression modulus $K_{\mathrm{nm}}$. This quantity is crucial in the description of properties of nuclei, supernovae explosions, neutron stars, and heavy ion collisions. Modern non-relativistic Hartree-Fock plus RPA calculations, using both Skyrme and Gogny effective interactions, indicate that the value of $K_{\mathrm{nm}}$ should be in the range 210-220 MeV [12]. In the framework of relativistic mean field theory on the other hand, calculations based on an effective interaction [10] with nuclear matter compression modulus $K_{\mathrm{nm}} \approx 250-270 \mathrm{MeV}$ are in better agreement with available data on spherical nuclei [1]. The complete experimental data set on isoscalar giant monopole resonances (GMR) has been recently analyzed by Shlomo and Youngblood [13]. In Fig. 1 we display results of time-dependent relativistic mean-field calculations of isoscalar and isovector oscillations in ${ }^{208} \mathrm{~Pb}$. The experimental isoscalar GMR energy in ${ }^{208} \mathrm{~Pb}$ is well established at $13.7 \pm 0.3 \mathrm{MeV}$. Experimental data on isovector giant monopole resonances are much less known. The systematics of excitation energies does not, in general, depend on the nuclear matter compression modulus. The experimental value for the isovector GMR in ${ }^{208} \mathrm{~Pb}: 26 \pm 3 \mathrm{MeV}$ [14]. We have calculated the ground-state with the NL1 [15] parameter set of the effective Lagrangian. This effective force has been extensively used in the description of properties of finite nuclei along the valley of beta stability [5]. For NL1 $\left(K_{\mathrm{nm}}=211.7 \mathrm{MeV}\right)$, we expect the calculated excitation energy for the isoscalar mode to be approximately 1-2 MeV lower than the average experimental value. However, the precise value is not important in the present consideration. For the initial deformation parameter in (14) we have used $a=0.2$. In the isoscalar case both proton and neutron densities are radially expanded, while for the isovector mode the proton density is initially 
compressed by the same amount. Therefore although the initial conditions are different, in both cases we follow the time evolution of the same system.

In Fig. 1a we plot the time history of the isoscalar monopole moment $\left\langle r^{2}(t)\right\rangle$, and in Fig. 1b the corresponding isovector moment is shown. The isoscalar mode displays regular undamped oscillations, while for the isovector mode we observe strongly damped anharmonic oscillations. On the right hand panels we plot the corresponding Fourier power spectra. The evolution time $T_{\text {final }}=3000 \mathrm{fm} / \mathrm{c}$ determines the numerical resolution $\Delta E=2 \pi \hbar c / T_{\text {final }}=\approx 0.4 \mathrm{MeV}$ in the frequency domain. The Fourier power spectra are calculated by FFT using data windowing with the Welch window function. We have verified that the positions of the main peaks do not depend on the choice of the window function. We estimate the numerical accuracy to $\approx 0.5 \mathrm{MeV}$. As one would expect for a heavy nucleus, there is very little spectral fragmentation in the isoscalar channel, and a single mode dominates at the excitation energy of $11 \mathrm{MeV}$. The Fourier spectrum of the isovector mode is strongly fragmented. However, the main peaks are found in the energy region $25-30 \mathrm{MeV}$, in agreement with the experimental data.

For the isoscalar mode, the time history of the monopole moment and the Fourier spectrum show that the oscillations of the collective coordinate are regular. On the other hand, the appearance of a broad spectrum of frequencies seems to indicate that the isovector oscillations are chaotic. A diagnosis of chaotic vibrations would imply that one has a clear definition of such motion. For a quantum system however, the concept of chaos, especially in time-dependent problems, is not well defined. And although our description of nuclear vibrations is semi-classical, quantum effects like the Pauli principle are present in the initial conditions and during the dynamical evolution. A number of diagnostic tests can help to identify chaotic oscillations in physical systems [16, and some of them can be applied in the present consideration. In Figs. 2 - 4 we display some additional qualitative 
measures which can be used to characterize the response of our nonlinear system. In Fig. 2 we have constructed the two-dimensional time-delayed pseudo-phase space for isoscalar (a), and isovector (b) oscillations shown in Fig. 1. Since information is available on the time evolution of just one variable, the collective coordinate, one plots the signal versus itself, but delayed or advanced by a fixed time constant $\left[\left\langle r^{2}(t)\right\rangle,\left\langle r^{2}(t+\tau)\right\rangle\right]$. The idea is that the signal $\left\langle r^{2}(t+\tau)>\right.$ is related to $\left\langle\dot{r}^{2}(t)>\right.$, and should have properties similar to those in the classical phase plane $\left[<r^{2}(t)>,<\dot{r}^{2}(t)>\right]$. The choice of the time delay $\tau$ is not crucial, except to avoid a natural period of the system. If the motion is chaotic, the trajectories in the phase space do not close. For the pseudo-phase planes shown in Fig. 2, we have taken $\tau=20 \mathrm{fm} / \mathrm{c}$. The phase space trajectories for the isoscalar mode are closed ellipses, indicating regular oscillations. For the isovector oscillations on the other hand, the trajectories are completely chaotic. The strong damping results from one-body processes: (i) escape of nucleons into the continuum states and (ii) collisions of the nucleons with the moving wall of the nuclear potential generated by the self-consistent mean fields. In Fig. 3 we display the corresponding Poincaré sections constructed from three-dimensional timedelayed pseudo-phase space $\left[\left\langle r^{2}(t)\right\rangle,\left\langle r^{2}(t+\tau)\right\rangle,\left\langle r^{2}(t+2 \tau)\right\rangle\right]$, with $\tau=20 \mathrm{fm} / \mathrm{c}$. The Poincaré maps are shown in the planes: $\left\langle r^{2}(t)\right\rangle=35 \mathrm{fm}^{2}$ for the isoscalar mode, and $\left\langle r^{2}(t)\right\rangle=-9.55 \mathrm{fm}^{2}$ for the isovector mode, respectively. The Poincaré map for the isoscalar mode consists of two sets of closely located points and therefore confirms regular oscillations. For the isovector oscillations the Poincaré map appears as a cloud of unorganized points in the phase plane. Such a map indicates stochastic motion. Another measure that is related to the Fourier transform is the autocorrelation function

$$
A(\tau)=\lim _{T \rightarrow \infty} \int_{0}^{T}<r^{2}(t)><r^{2}(t+\tau)>d t
$$

When the signal is chaotic, information about its past origins is lost. This means that 
$A(\tau) \rightarrow 0$ as $\tau \rightarrow \infty$, or the signal is only correlated with its recent past. It is also expected $A(\tau)$ of a chaotically modulated signal to be an irregularly modulated waveform. The autocorrelation functions for isoscalar and isovector oscillations are shown in Fig. 4. The normalization is $A(\tau=0)=1$. For the isovector mode $A(\tau)$ shows a rapid decrease and the envelope appears as an irregular waveform. Therefore also this quantity indicates that the dynamical variable displays chaotic oscillations for the isovector mode.

In our first example we have considered monopole oscillations in ${ }^{208} \mathrm{~Pb}$, for which there exist experimental data on both isoscalar and isovector giant monopole resonances. Thus our description of the dynamics of monopole oscillations is not just a completely artificial model, but in fact corresponds to an experimentally observed physical system. On the other hand ${ }^{208} \mathrm{~Pb}$ is a large and complicated system, in which many single-nucleon orbitals contribute to the collective coordinate. In order to study in more detail the dynamics, it is more convenient to consider a light nucleus: ${ }^{16} \mathrm{O}$. In Fig. 5 we compare the isoscalar and isovector monopole moments and the associated Fourier power spectra. For the isoscalar mode a modulation of the signal is observed, and two strong peaks at approximately $20 \mathrm{MeV}$ excitation energy are found in the Fourier spectrum. A fragmentation in the Fourier spectrum is expected for such a light nucleus. The behavior of the collective coordinate for the isovector mode appears to be chaotic. The Fourier spectrum is strongly fragmented in the energy region $15-45 \mathrm{MeV}$. The pseudo-phase spaces are compared in Fig. 6. The results are similar to those for ${ }^{208} \mathrm{~Pb}$ and indicate that isovector oscillations are chaotic.

In Refs. [17, 18] Blocki et al. analyzed the behavior of a purely classical gas of noninteracting point particles enclosed in a hard-wall container, which undergoes periodic and adiabatic shape oscillations. The wall motion is not modified by the collisions with the particles, i.e. the dynamics is not self-consistent. Analyses of Poincaré sections 
and of Lyapunov exponents showed that for low deformations $(l=2)$ the motion of particles is regular, whereas for higher multipolarities $(l \geq 3)$ the scattering of segments of wall with positive curvature leads to divergence between trajectories and therefore to chaotic motion. A somewhat different result, especially if applied to the nuclear system, was found in Ref. [19]. Conditions under which nucleons inside a nucleus can undergo chaotic motion were studied. A self-consistent model of separable forces was used in a semi classical approximation of the time-dependent Hartree-Fock equation. The test particle method was used to solve the Vlasov equation for the time-evolution of the density matrix. Isoscalar quadrupole and octupole oscillations were investigated. It was shown that, both for quadrupole and octupole deformations, chaotic single-particle dynamics leads to regular motion of the collective coordinate, the multipole moment of deformation. The origin of the chaotic single-particle dynamics was attributed to the exchange of energy between the motion of the individual test particles and the collective motion of the multipole coordinate. In particular, it was stressed that self-consistency is essential for the generation of regular dynamics from an ensemble of single-nucleons with chaotic trajectories. The relationship between chaoticity at the microscopic level and dissipation of the collective degrees of freedom was also investigated in Ref. [20]. Classical particles were confined in a two-dimensional nuclear potential whose walls undergo periodic shape oscillations of fixed multipolarity. The collective variable appears explicitly in the Hamiltonian as an additional degree of freedom. A fully self-consistent description of the dynamics of particle motion and the collective coordinate was employed. Isoscalar monopole oscillations were studied and it was shown that self-consistency induces chaotic single-particle motion. In particular, it was demonstrated how the coupling between the collective variable and single-particle dynamics induces macroscopic dissipation.

In a quantum description of nuclear dynamics, such as the one based on time- 
dependent relativistic mean-field theory, we cannot follow the trajectories of individual nucleons. In order to better understand the difference between the isoscalar and isovector modes at the microscopic level, we consider the individual contributions of single-nucleon orbitals to the collective coordinate, the time-dependent monopole moment. In the case of ${ }^{16} \mathrm{O}(Z=N=8)$, protons and neutrons occupy only three spherical levels in the ground state: $1 s 1 / 2,1 p 3 / 2$, and $1 p 1 / 2$. Monopole oscillations do not break the degeneracy, i.e. spherical symmetry is conserved during the time evolution of the nuclear system and we can follow the dynamics of each level. In Fig. 7 we display the time histories of the expectation values of $r^{2}$ for the three neutron levels. In Fig. 7a we plot $\left\langle r^{2}(t)>_{s 1 / 2, p 3 / 2, p 1 / 2}\right.$ for the isoscalar mode, for which the oscillations of the collective coordinate are shown in Fig. 5a. Fig. 7b corresponds to the isovector monopole oscillations displayed in Fig. 5b.

In contrast to the collective isoscalar and isovector moments shown in Fig. 5, in Fig. $7 \mathrm{a}$ and $7 \mathrm{~b}$ we plot the same quantities, expectation values of $r^{2}$ for the neutron singleparticle orbitals. Also the initial conditions for neutron motion are the same in both cases. The only difference is the initial condition for protons. In the isoscalar case protons oscillate in phase with neutrons. Proton and neutron oscillations are out of phase for the isovector mode. In Fig. 7a one observes regular modulated oscillations for all three neutron levels. For the isovector case the time histories display chaotic oscillations. In Fig. 8 we display the corresponding Fourier power spectra and the largest Lyapunov exponents. Comparing the Fourier spectra, we notice that the differences between isoscalar and isovector oscillations are especially pronounced for the $1 s$ 1/2 level. Much more fragmentation is observed in the isovector spectrum, and the strength is shifted toward higher frequencies. The Fourier spectra for isovector oscillations display pronounced peaks in the higher frequency region. From the time series of the expectation values $\left\langle r^{2}(t)\right\rangle_{s 1 / 2, p 3 / 2, p 1 / 2}$, we have also calculated the largest Lyapunov exponents, shown in the right hand panels 
of Fig. 8. Lyapunov exponents provide a qualitative and quantitative characterization of dynamical behavior. They are related to the exponentially fast divergence or convergence of nearby orbits in phase space. A system with one or more positive Lyapunov exponents is defined to be chaotic. The magnitude of the exponents reflects the time scale on which system dynamics becomes unpredictable. The largest exponents are displayed as function of evolution time in Fig. 9. They have been calculated by the method of Ref. [21], which allows the estimation of non-negative Lyapunov exponents from a time series. For the $1 s 1 / 2$ level the calculated exponent for oscillations that correspond to the isoscalar collective mode is consistent with the value zero, indicating regular motion. For the isovector mode, the Lyapunov exponent is positive and large. Therefore, oscillations of $\left\langle r^{2}(t)\right\rangle_{s 1 / 2}$ are chaotic for collective isovector vibrations. For the $1 p 3 / 2$ and $1 p 1 / 2$ levels all calculated exponents are positive. For the isoscalar mode the small positive values reflect the observed fragmentation in the Fourier spectrum. Those that correspond to isovector collective mode are much larger and indicate that the dynamics is indeed chaotic. The corresponding Fourier spectra and largest Lyapunov exponents for proton levels are very similar. The only difference is that, in addition to meson exchange forces, the protons also feel the long range Coulomb interaction.

Similar results are obtained for ${ }^{208} \mathrm{~Pb}$. We have calculated the Fourier spectra and largest Lyapunov exponents for the time series of expectation values $\left\langle r^{2}>\right.$ for the 22 single-particle spherical shell-model neutron states. Typical results are shown in Fig. 9 for the orbitals $1 h 11 / 2,1 h 9 / 2$, and $2 f 7 / 2$. The column on the left corresponds to isoscalar monopole collective oscillations, on the central panels the results for the isovector collective mode are shown. Both for isoscalar and isovector oscillations (Fig. 1), the initial conditions for neutron motion are the same. All calculated Lyapunov exponents are positive, and therefore seem to indicate that the underlying microscopic dynamics 
is chaotic. In general, we observe much more fragmentation in the Fourier spectra of oscillations that correspond to the isovector mode, and the resulting Lyapunov exponents have also larger values. The interesting result of course is that for the isoscalar case we observe regular oscillations of the collective variable. This would be in agreement with the results reported in Ref. [19], where chaotic single-particle motion was found in coexistence with regular collective dynamics. For the isovector mode on the other hand, the collective monopole moment displays chaotic oscillations. This is explained by the fact that protons and neutrons effectively move in two self-consistent potentials that oscillate out of phase. For example, when neutrons move inward, they scatter on the potential wall with positive curvature that is created by protons moving outward. This will lead to pseudo-random motion of the nucleons and dissipation of collective oscillations.

\section{Conclusions}

Isoscalar and isovector collective monopole oscillations in spherical nuclei have been analyzed within the framework of time-dependent relativistic mean field theory. These oscillations correspond to the most elementary collective nuclear modes, the giant resonances. In order to investigate the dynamics of collective vibrations, we have analyzed time-dependent and self-consistent calculations that reproduce the experimental data on monopole giant resonances in spherical nuclei. Because of the self-consistent time-evolution, the nuclear system is non-linear and one expects chaotic dynamics for specific initial conditions. In particular, we have studied the difference in the dynamics of isoscalar and isovector collective modes. Time histories, Fourier spectra, state-space plots, Poincaré sections, autocorrelation functions, and Lyapunov exponents have been used to characterize the nonlinear system and to identify chaotic oscillations. It has been

shown that the oscillations of the collective coordinate can be characterized as regular 
for the isoscalar mode, and that they become chaotic when initial conditions correspond to the isovector mode. Our results also confirm the conclusions of a number of studies, which have shown how a regular collective mode can coexist with chaotic single-particle dynamics. However, we have shown that this is the case only for isoscalar modes, that is, only if one considers the motion of a single type of particles. When protons and neutrons move out of phase, as it happens for isovector modes, the resulting dynamics of the collective coordinate exhibit chaotic behavior. Of course, analogous considerations apply also for higher multipolarities, for example isoscalar and isovector giant quadrupole resonances. Due to numerical problems, for quadrupole oscillations our computer codes could not propagate the nuclear system to very long times. However, a preliminary analysis of isoscalar and isovector quadrupole oscillations in ${ }^{16} \mathrm{O}\left(T_{\text {final }}=500 \mathrm{fm} / \mathrm{c}\right)$ have shown that results similar to the monopole resonances could be expected. Work along these lines is in progress. 


\section{Acknowledgments}

This work has been supported in part by the Bundesministerium für Bildung und Forschung under contract 06 TM 875.

\section{References}

[1] V. Zelevinsky; Annu. Rev. Nucl. Part. Sci. 46 (1996) 237

[2] B.D. Serot and J.D. Walecka; Adv. Nucl. Phys. 16 (1986) 1

[3] P.G. Reinhard; Rep. Prog. Phys. 52 (1989) 439

[4] B.D. Serot; Rep. Prog. Phys. 55 (1992) 1855

[5] P. Ring; Progr. Part. Nucl. Phys. 37 (1996) 193

[6] D. Vretenar, H. Berghammer, and P. Ring; Phys. Lett. B319 (1993) 29

[7] D. Vretenar, H. Berghammer, and P. Ring; Nucl. Phys. A581 (1995) 679

[8] P. Ring, D. Vretenar, and B. Podobnik; Nucl. Phys. A598 (1996) 107

[9] B. Podobnik, D. Vretenar, and P. Ring; Z. Phys. A354 (1996) 375

[10] G. A. Lalazissis, J. König and P. Ring; Phys. Rev. C 55 (1997) 540

[11] D. Vretenar, G. A. Lalazissis, R. Behnsch, W. Pöschl and P. Ring; Nucl. Phys. A (1997)

[12] J.P. Blaizot, J.F. Berger, J. Dechargé, and M. Girod; Nucl. Phys. A591 (1995) 435

[13] S. Shlomo and D.H. Youngblood; Phys. Rev. C47 (1993) 52 
[14] P. Gleissl, M. Brack, J. Meyer, and P. Quentin; Ann. Phys. (N.Y.) 197 (1990) 205

[15] P.G. Reinhard, M. Rufa, J. Maruhn, W. Greiner, and J. Friedrich; Z. Phys. A323 (1986) 13

[16] A. H. Nayfeh and B. Balachandran; Applied Nonlinear Dynamics, John Wiley, New York (1995)

[17] J. Blocki, F. Brut, T. Srokowski, and W. J. Swiatecki; Nucl. Phys. A545 (1992) $511 \mathrm{c}$

[18] J. Blocki, J. J. Shi, and W. J. Swiatecki; Nucl. Phys. A554 (1993) 387

[19] W. Bauer, D. McGrew, V. Zelevinsky, and P. Schuck; Phys. Rev. Lett. 72 (1994) 3771

[20] G. F. Burgio, M. Baldo, A. Rapisarda, and P. Schuck; Phys. Rev. C 52 (1995) 2475

[21] A. Wolf, J. B. Swift, H. L. Swinney, and A. Vastano; Physica 16D (1985) 285 


\section{Figure Captions}

Fig. 1 Time-dependent isoscalar $<r^{2}>$ (a) and isovector $<r_{\mathrm{p}}^{2}>-<r_{\mathrm{n}}^{2}>$ (b) monopole moments and the corresponding Fourier power spectra for ${ }^{208} \mathrm{~Pb}$. The parameter set of the effective Lagrangian is NL1.

Fig. 2 Two-dimensional pseudo-phase space for isoscalar (a) and isovector (b) monopole oscillations in ${ }^{208} \mathrm{~Pb}$.

Fig. 3 Poincaré sections for isoscalar (a) and isovector (b) monopole oscillations in ${ }^{208} \mathrm{~Pb}$.

Fig. 4 Autocorrelation functions for isoscalar (a) and isovector (b) monopole oscillations in ${ }^{208} \mathrm{~Pb}$.

Fig. 5 Time-dependent isoscalar (a) and isovector (b) monopole moments and the Fourier spectra for ${ }^{16} \mathrm{O}$. The effective force is NL1.

Fig. 6 Two-dimensional pseudo-phase space for isoscalar (a) and isovector (b) monopole oscillations in ${ }^{16} \mathrm{O}$.

Fig. 7 Time-dependent expectation values $\left\langle r^{2}\right\rangle$ for the three single neutron spherical shell model states $1 s 1 / 2,1 p 3 / 2$, and $1 p 1 / 2$ in ${ }^{16} \mathrm{O}$. (a) corresponds to the isoscalar oscillations of the collective coordinate. In panel (b) results for the isovector collective mode are shown.

Fig. 8 Fourier spectra and the largest Lyapunov exponents for the time-dependent expectation values $<r^{2}>$ in the three single neutron spherical shell model states $1 s 1 / 2,1 p 3 / 2$, and $1 p 1 / 2$ in ${ }^{16} \mathrm{O}$. Dashed lines indicate the isoscalar collective monopole mode, solid lines correspond to isovector collective oscillations. 
Fig. 9 Fourier spectra and the largest Lyapunov exponents for the time-dependent expectation values $<r^{2}>$ in the $1 h 11 / 2,1 h 9 / 2$, and $2 f 7 / 2$ single neutron spherical shell model states in ${ }^{208} \mathrm{~Pb}$. Results that correspond to the isoscalar collective monopole mode are shown on the left, those for the isovector mode are in the center. Lyapunov exponents are displayed on right hand side panels. 

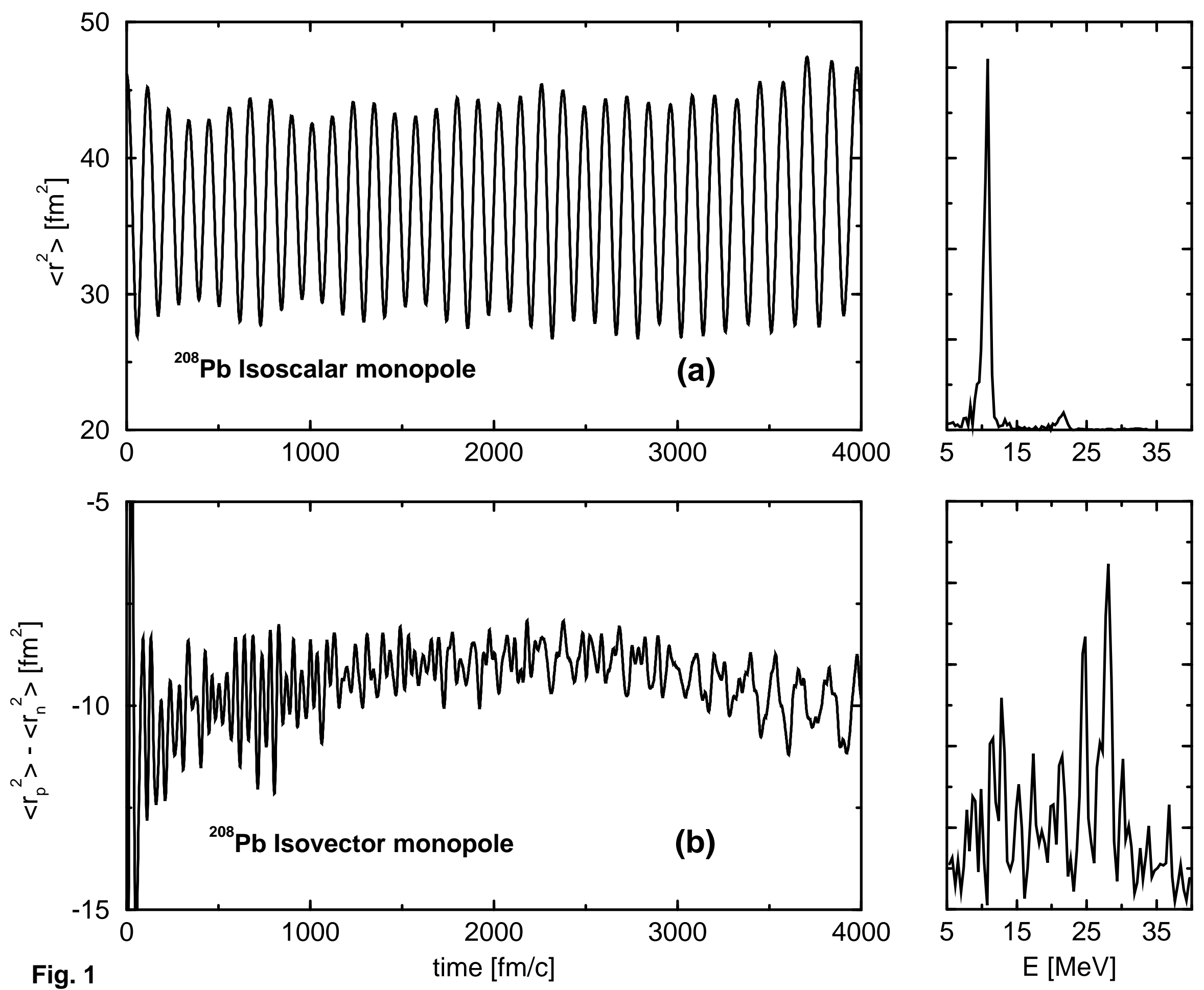

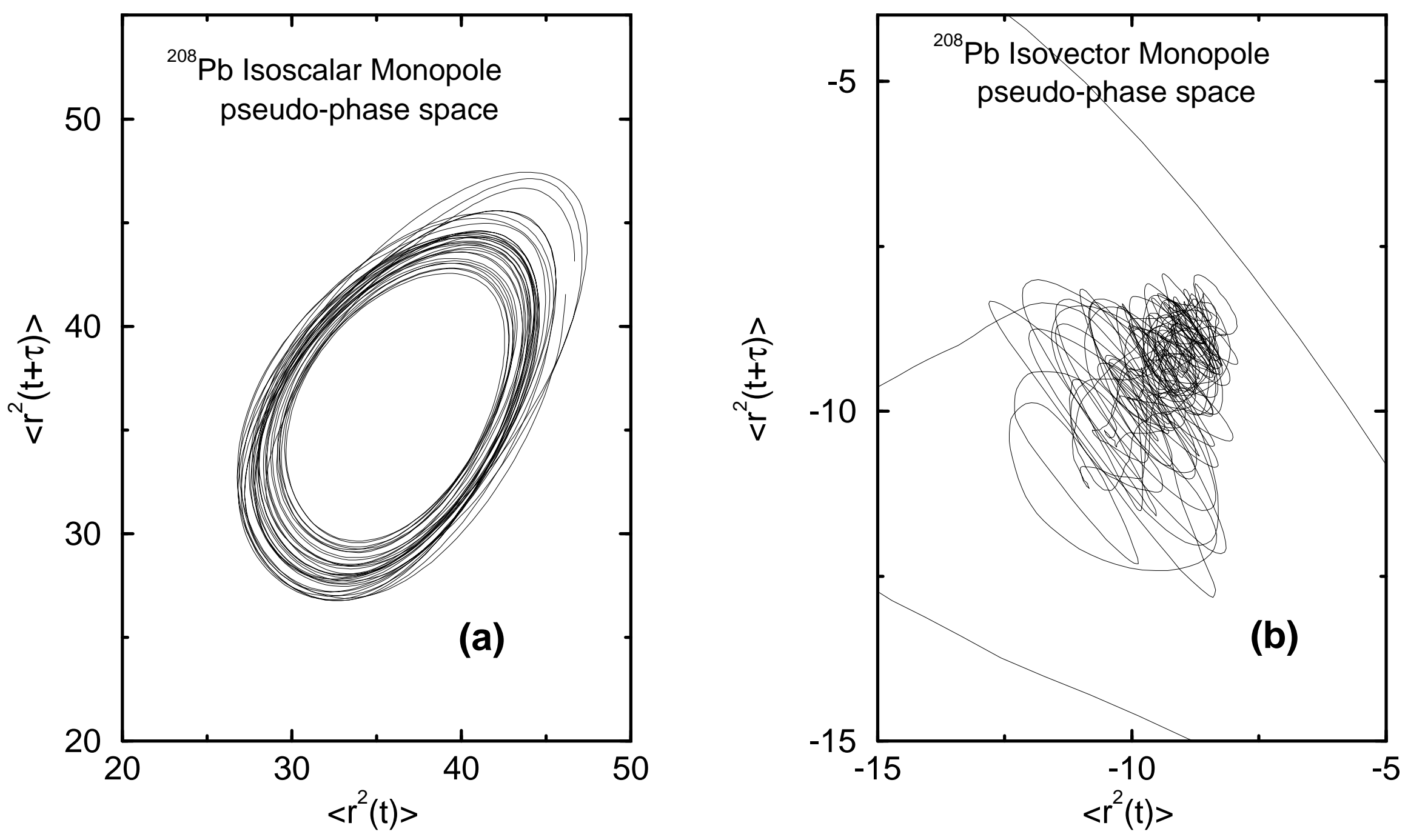

Fig. 2 

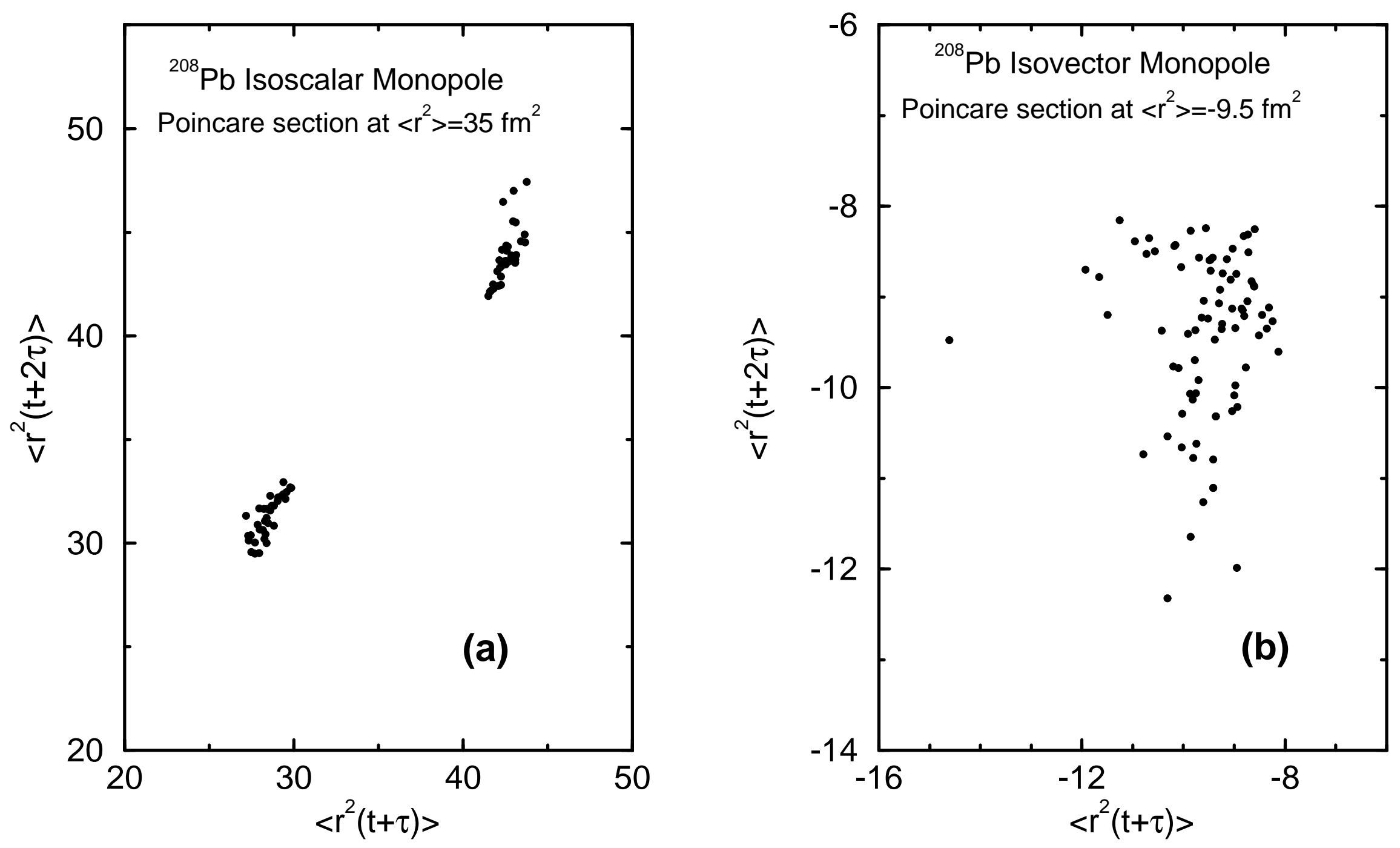

Fig. 3 

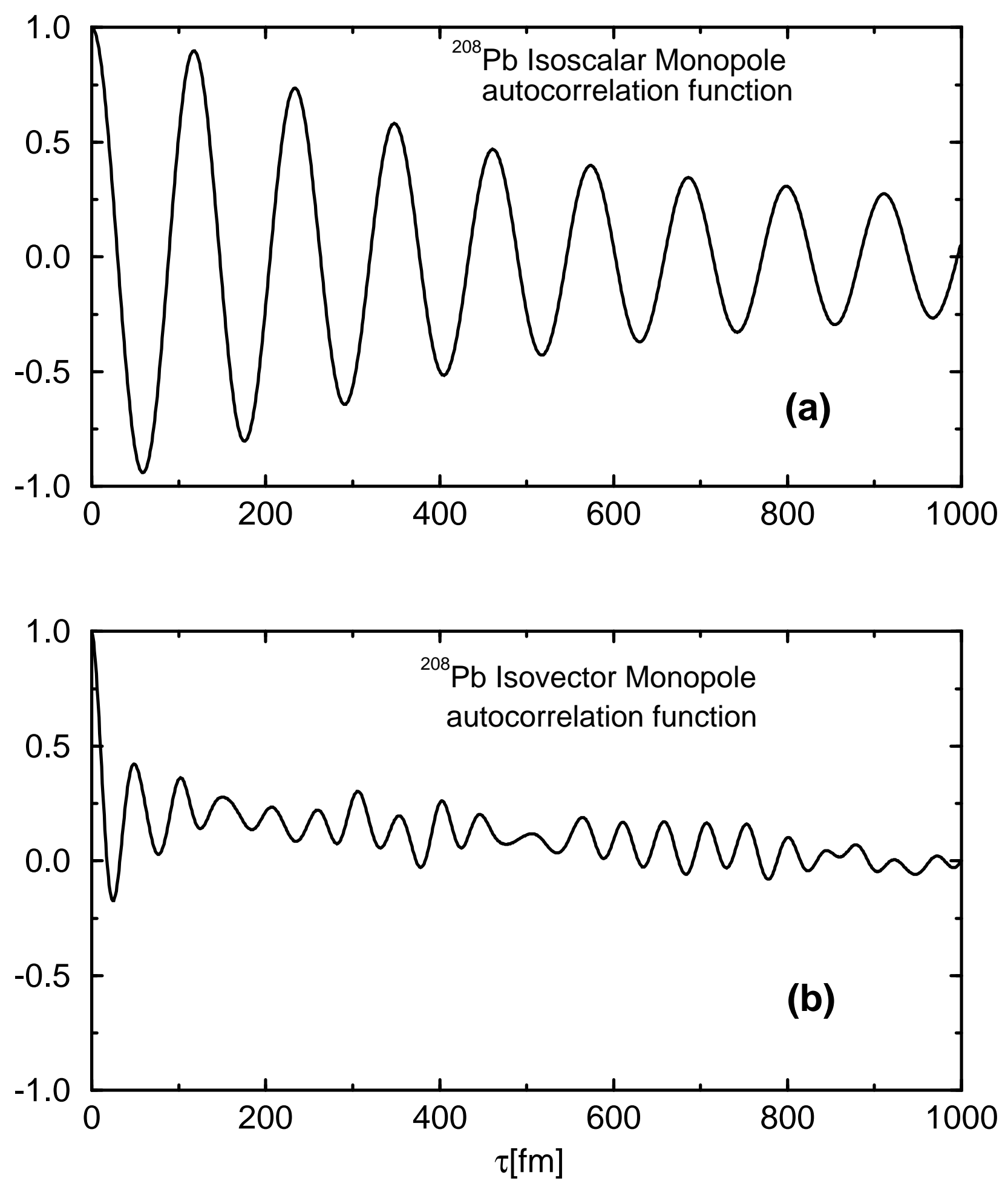

Fig. 4 

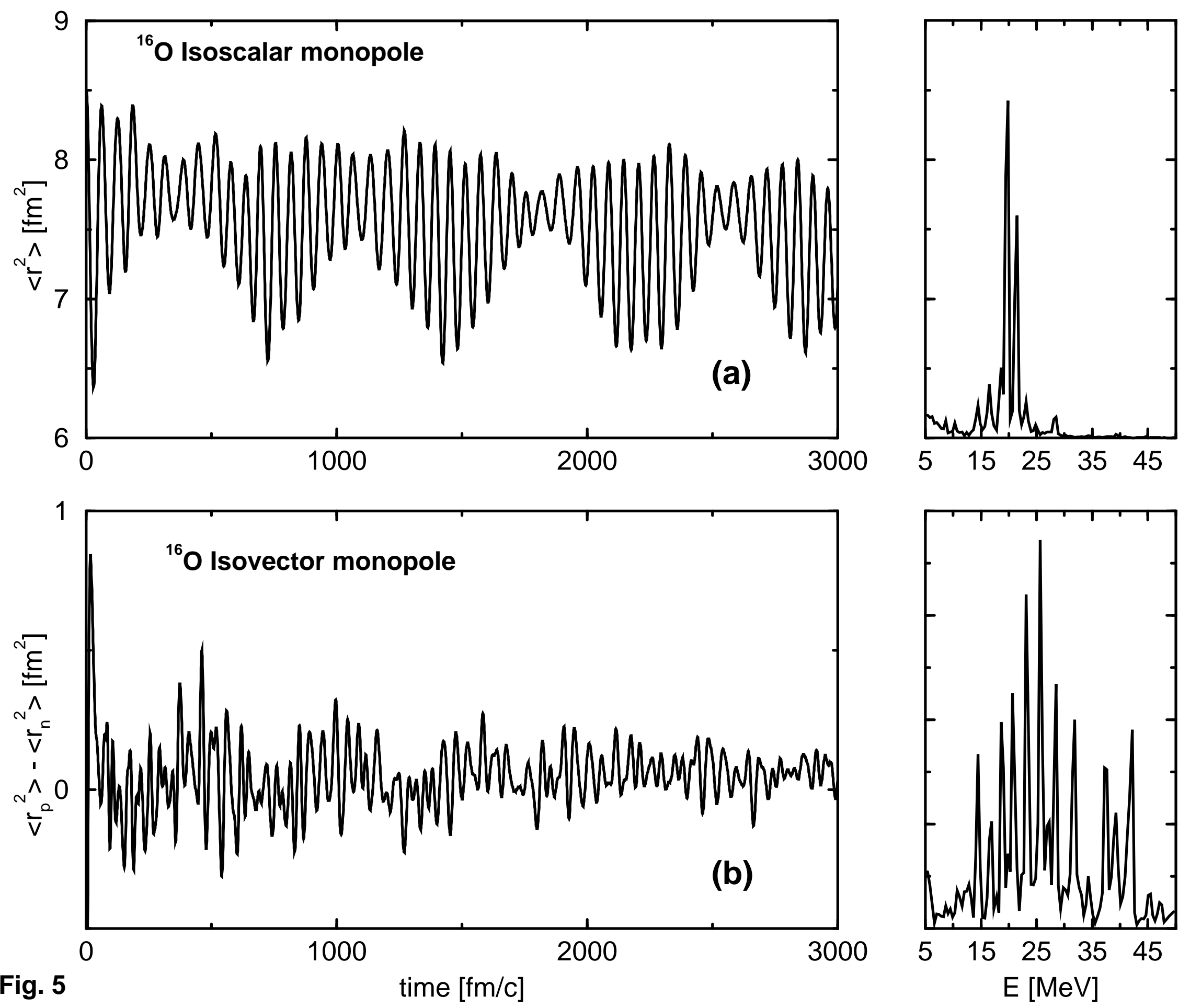

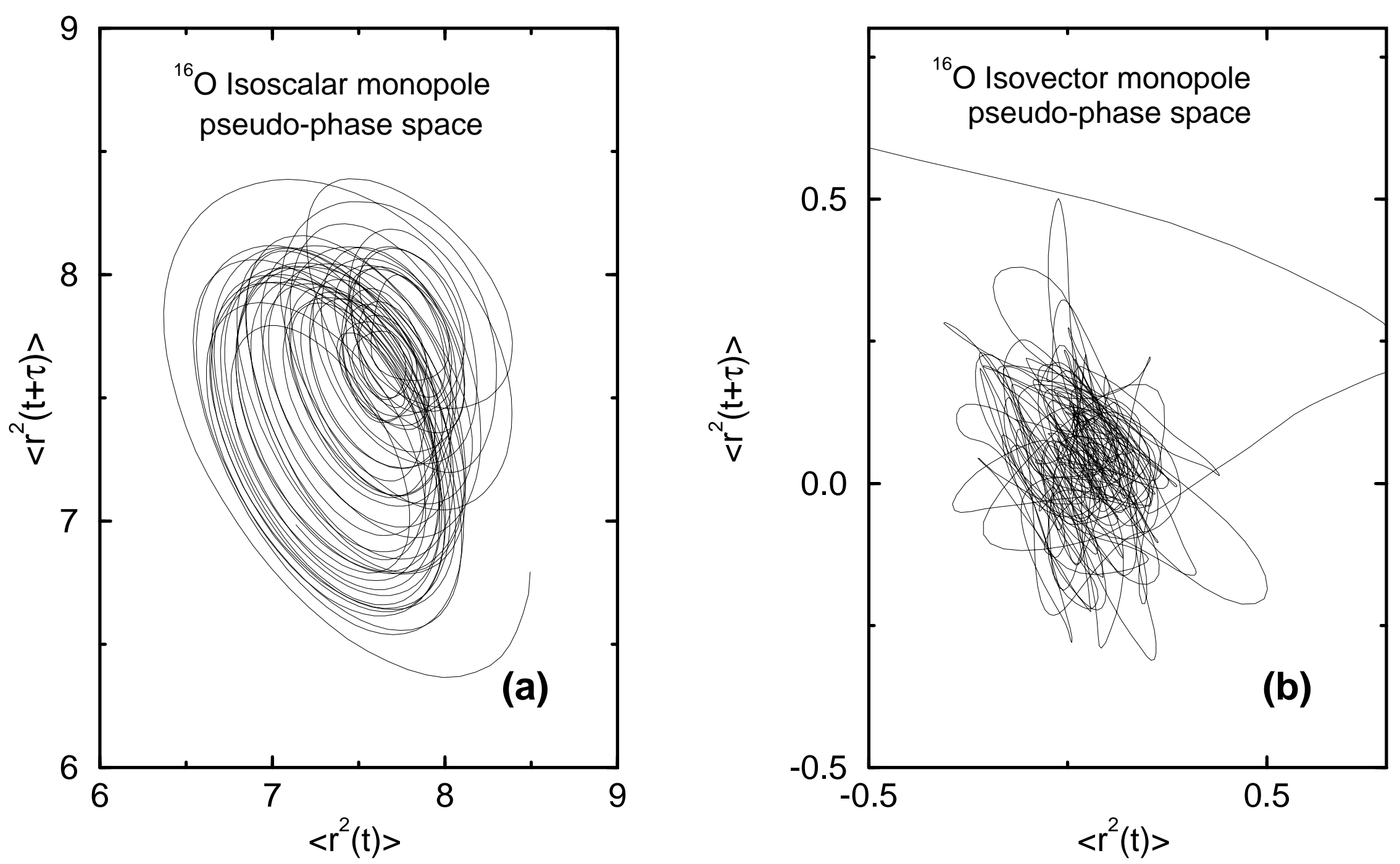

Fig. 6 

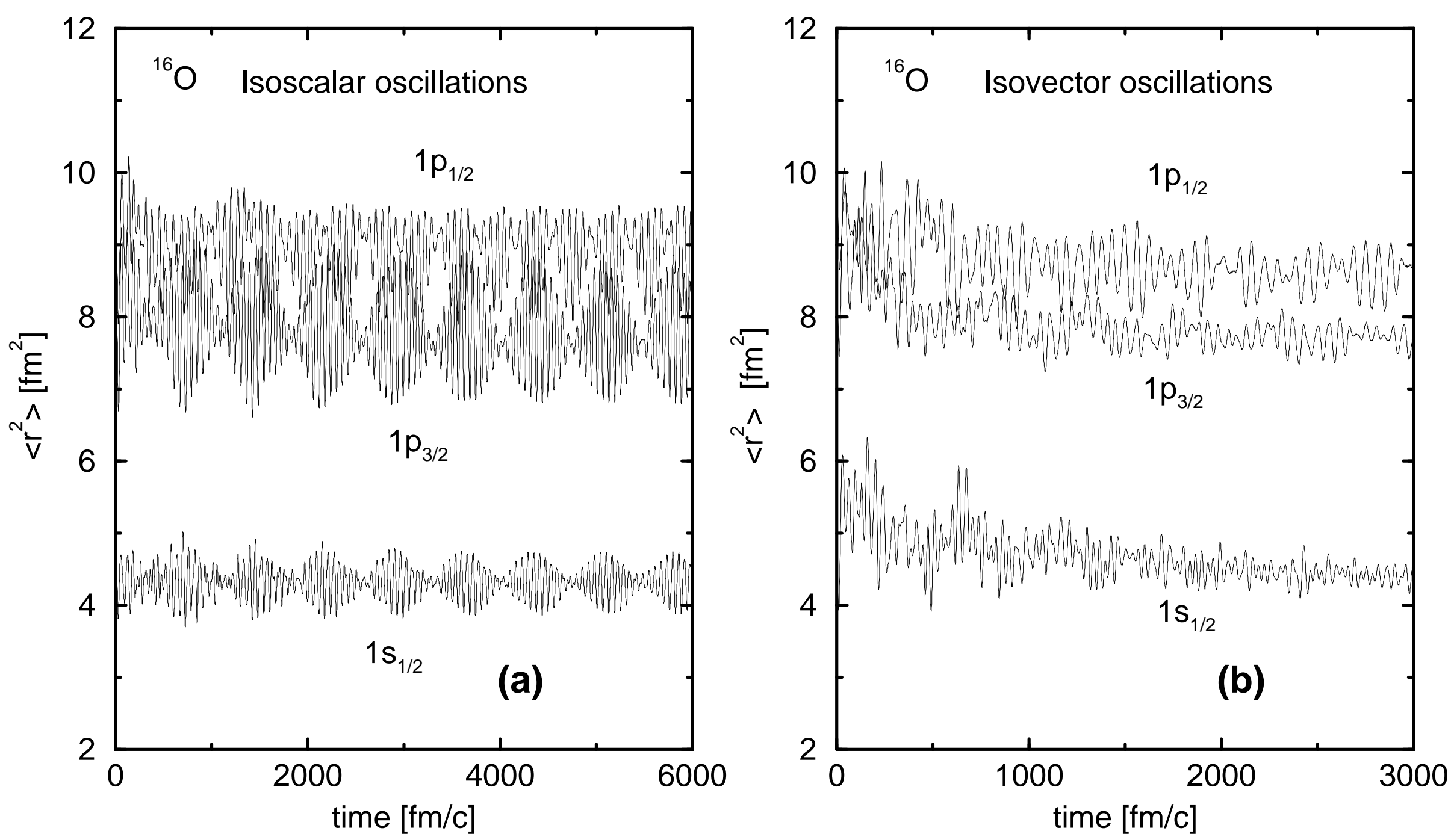

Fig. 7 

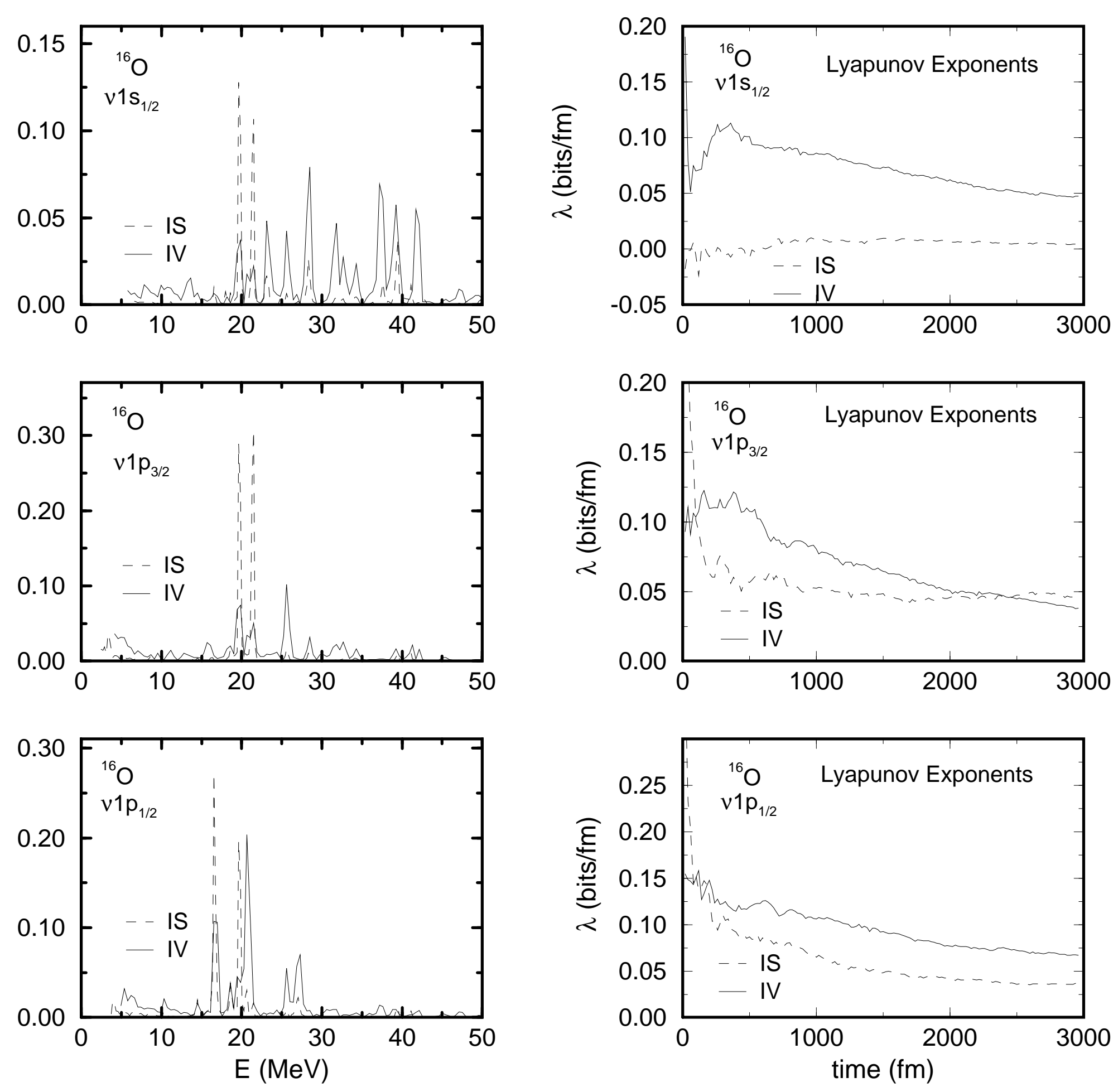

Fig. 8 

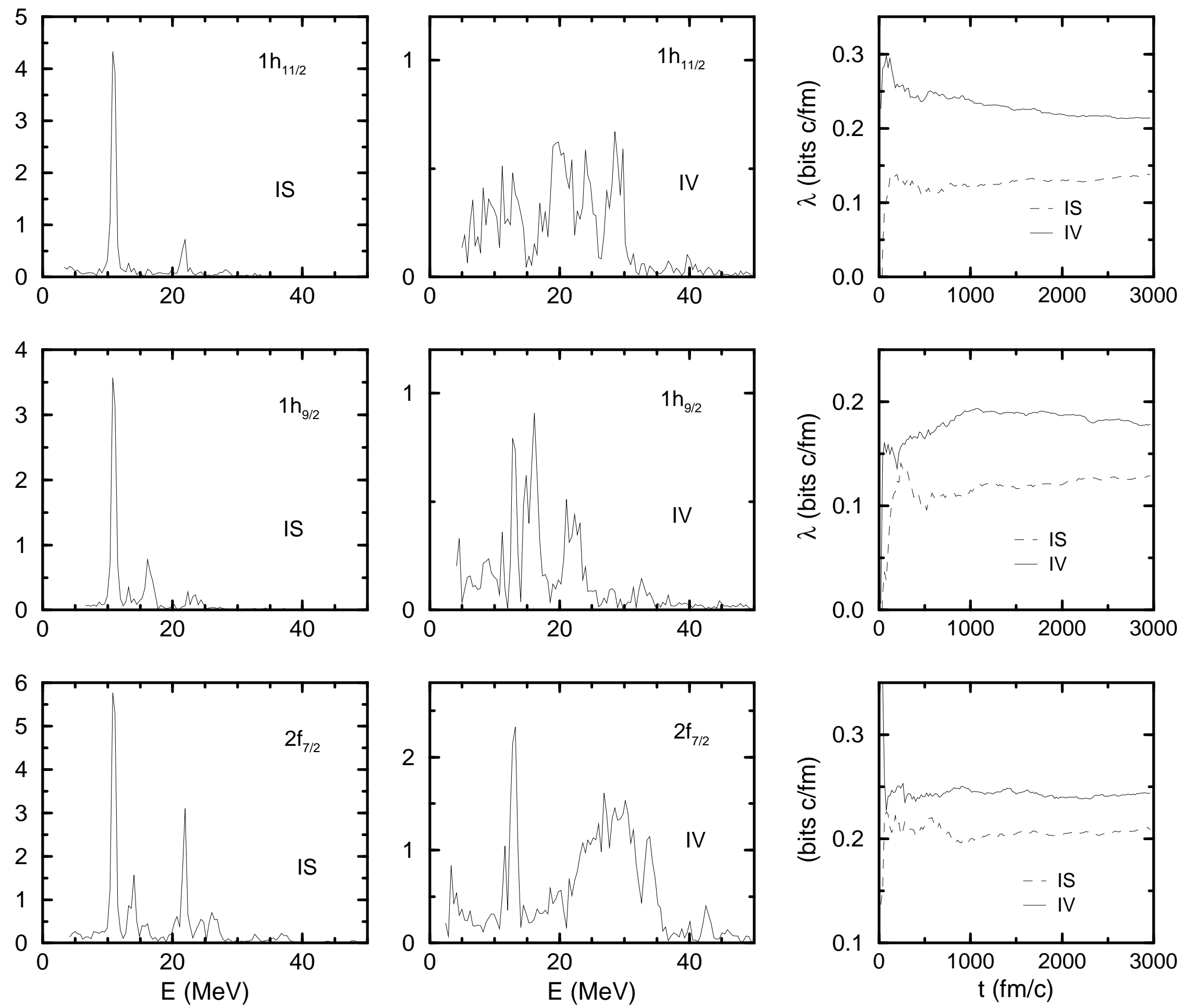

Fig. 9 